\title{
Expanding the clinical potential of cardiac radionuclide adrenergic imaging
}

\author{
Mark I. Travin, MD, MASNC ${ }^{a}$ \\ a Division of Nuclear Medicine, Department of Radiology, Montefiore Medical Center and the \\ Albert Einstein College of Medicine, Bronx, NY
}

Received Jan 21, 2019; accepted Jan 22, 2019

doi: $10.1007 / \mathrm{s} 12350-019-01629-8$

\section{See related article, pp. 2202-2209}

The autonomic nervous system, divided into sympathetic and parasympathetic components, plays an essential role in sustaining body functions. In particular, direct cardiac autonomic innervation working in conjunction with circulating neurohormones (epinephrine, norepinephrine (NE), renin-angiotensin-aldosterone system (RAAS) hormones, cytokines) controls cardiac function (heart rate, contractility) and adjusts vascular tone for maintenance of proper perfusion to organs and tissues. ${ }^{1}$

There is a long history of exploring methods to assess human autonomic nervous system activity. These include efforts to measure the sympathetic component through determination of systemic plasma concentration, organ-specific amounts, and regional spillover of the chemical mediator norepinephrine $(\mathrm{NE}){ }^{2}$ One can also perform direct measurements of systemic postganglionic sympathetic activity via microneurography that records pulses in a peripheral nerve ${ }^{3}$ commonly the peroneal. Alternatively, for focused assessment of cardiac sympathetic activity, one can directly visualize and quantitate cardiac uptake and washout of catecholamines using radionuclide tracer analogues of NE, such as SPECT iodine-123 meta-iodobenzylguanidine $\left({ }^{123} \mathrm{I}-\right.$ $m \mathrm{IBG})^{4}$ and various positron emission tomographic (PET) agents, ${ }^{5}$ which are taken up and concentrated in cardiac innervating sympathetic nerves. Most clinical

Reprint requests: Mark I. Travin, MD, MASNC, Division of Nuclear Medicine, Department of Radiology, Montefiore Medical Center and the Albert Einstein College of Medicine, 111 E. 210th Street, Bronx, NY 10467-2490; mtravin@attglobal.net

J Nucl Cardiol 2020;27:2210-5.

1071-3581/\$34.00

Copyright (C) 2019 American Society of Nuclear Cardiology. focus has been on ${ }^{123} \mathrm{I}-m \mathrm{IBG}$, with studies showing convincing evidence that such imaging is effective in risk stratifying patients with advanced heart failure (HF) independently of, and often better than, conventional techniques including measurement of plasma NE levels, ${ }^{6}$ and is also potentially useful in evaluating other cardiac conditions such as arrhythmias and ischemic heart disease. ${ }^{4}$

Of course, a crucial objective of cardiac risk stratification with ${ }^{123} \mathrm{I}-m \mathrm{IBG}$ and analogous PET adrenergic tracers is to effectively guide therapies that can reduce mortality and improve the quality of life. While there is a strong logical basis and robust observational data demonstrating that adrenergic imaging can, beyond and arguably better than commonly used methods, help guide the use of invasive expensive cardiac electronic devices and procedures, no one has yet been able to undertake and complete the necessary prospective randomized clinical studies needed for inclusion of such imaging into societal guidelines and use in routine clinical practice. Consequently, and also because of financial constraints, after decades of investigation and discussions about this relatively easy, noninvasive technique that assesses a crucial component of cardiovascular function in health and disease, it is not being used. $^{7}$

\section{POTENTIAL TO BROADEN THE POTENTIAL USE OF CARDIAC ADRENERGIC IMAGING BY CONSIDERING ASSOCIATION OF CARDIAC DISEASE WITH OTHER ORGAN SYSTEMS}

As sympathetic innervation involves the whole body, clinical use of sympathetic cardiac imaging could be encouraged by finding utility in performing it in conjunction with diseases related to other systems and organs. For example, there is currently much interest in cardiac ${ }^{123} \mathrm{I}-m \mathrm{IBG}$ imaging to more accurately distinguish Parkinson's disease and dementia with Lewy 
bodies from other similar appearing neurologic conditions. $^{7}$

However, a key organ that interacts closely with the heart, and that is highly controlled by autonomic innervation, is the kidney. Renal sympathetic efferent and afferent neurons innervate the kidneys via their course within and just adjacent to the renal artery wall, proceeding to renal vasculature, tubules, and the juxtaglomerular apparatus, serving to regulate volume retention through sodium reabsorption, control renal blood flow via neutrally mediated effects on local vascular tone, and stimulate renin release that directs the renin-angiotensin-aldosterone system (RAAS), while also sending afferent signals to the brain that in turn sends out efferent signals adjusting cardiac function and systemic vascular tone. ${ }^{8}$ Under normal physiologic conditions, this interplay between the heart and kidney acts in concert to maintain proper homeostasis at rest and during various physiologic "stresses." However, in pathologic conditions such as hypertension, systemic, renal, and cardiac sympathetic tone is abnormal. The abnormality is manifest systemically as increased muscle sympathetic nerve activity (MSNA), increased plasma NE levels, and higher NE plasma spillover from activated sympathetic fibers, ${ }^{1}$ while locally at the kidney there is increased NE regional spillover and an increase of renal sympathetic nerve firing. ${ }^{9}$ Augmented cardiac and vascular sympathetic nerve activity is demonstrated by increased cardiac NE spillover, a higher rate of left ventricular ejection, reduced aortic compliance, and arteriolar vasoconstriction. $^{2,8}$

In response to the aforementioned understanding of the role of renal sympathetic innervation in the pathophysiology of hypertension, as early as the 1950s surgical disruption of renal sympathetic innervation has been undertaken in an attempt to treat resistant disease, but this approach has been associated with prohibitively high morbidity, mortality, and long-term complications. ${ }^{10} \mathrm{~A}$ major breakthrough was the development of a percutaneous approach in which a catheter is threaded into the renal arteries, with subsequent ablation of the nearby sympathetic nerves using radiofrequency energy or more recently ultrasound. ${ }^{11}$ Among the first to demonstrate the efficacy of catheterbased renal sympathetic denervation (RDN) was Krum et al., ${ }^{10}$ who reported a substantial and sustained reduction in blood pressure (BP) without serious adverse events. Subsequent work has shown that catheter-based RDN not only reduces renal NE spillover, a manifestation of blockage of renal efferent sympathetic activity that leads to turning-off of the renin-angiotensin-aldosterone system, but also produces a sustained reduction in muscle sympathetic nerve pulses not directly related to the $\mathrm{BP}$ reduction, ${ }^{12}$ and therefore likely to involve alterations in afferent signals from the kidney that are relayed to integrative structures in the brainstem and hypothalamus with resultant suppression of central sympathetic outflow. ${ }^{8}$

Interesting insights into the potential for RDN to affect cardiac pathophysiology were reported in a study by Brandt et al. ${ }^{13}$ in which patients with medicationresistant hypertension underwent RDN. Six months after the procedure, not only was there a significant reduction in BP, but also reductions in intraventricular septal thickness and left ventricular mass index, improved diastolic function with increased diastolic relaxation velocity and decreased filling pressures, and a significant increase in ejection fraction. A key finding was that left ventricular hypertrophy regression was not exclusively associated with BP reduction, raising speculation that decreases in systemic sympathetic tone, with reduced RAAS activity, facilitated regression of myocardial fibrosis beyond that from the BP decrease.

\section{HOW MIGHT MIBG IMAGE FINDINGS RELATE TO ALTERATIONS IN SYSTEMIC SYMPATHETIC ALTERATIONS?}

Given these demonstrated effects of RDN on the heart, one would expect that cardiac ${ }^{123} \mathrm{I}-m \mathrm{IBG}$ imaging could be an effective way to assess the results of the procedure. Nevertheless, one must consider that the relationship of image findings to RDN is likely to be complex. For example, not only would changes in systemic sympathetic input to the heart influence cardiac ${ }^{123} \mathrm{I}-m$ IBG uptake, but pathologic structural and functional changes to the myocardium and innervating sympathetic neurons would also affect ${ }^{123} \mathrm{I}-m \mathrm{IBG}$ images.

Cardiac sympathetic innervation is most commonly assessed by global tracer uptake on a planar image in relation to mediastinal background, i.e., the heart-tomediastinum ratio (HMR), found most useful in delayed images that reflect tracer retention. While there is robust evidence that a reduced HMR is associated with a variety of worsened cardiac outcomes, there are several mechanisms that can result in this image finding. As described by Fallavollita and Canty, ${ }^{14}$ a reduced HMR can indicate either "denervation", from anatomic loss of sympathetic nerves, or "dysinnervation", in which an insult results in sympathetic nerve dysfunction that can be reversible with recovery from injury. The dysfunction can involve abnormalities in any of several components of tracer uptake and storage in the pre-synaptic sympathetic terminal, including disruption or downregulation of the NET uptake-1 transporter, problems with the vesicular monoamine transport (VMAT) of tracer into protective vesicles, issues related to the degradative 
monoamine oxidase (MAO) enzyme, and perhaps also increased extraneuronal uptake (uptake 2). ${ }^{15}$

Another matter to consider is that the relation of cardiac MIBG uptake to the systemic sympathetic state is unclear. While a correlation between the HMR and myocardial NE content has been shown, in a study of patients with cardiomyopathy, both Schofer et al. ${ }^{16}$ and Tamaki et al. ${ }^{17}$ did not find significant correlations between HMR and plasma catecholamine levels.

Another commonly measured parameter in cardiac ${ }^{123} \mathrm{I}-m \mathrm{IBG}$ imaging is tracer decrease between early (about 15 minutes after tracer administration) and delayed (approximately 4 hour later) images, i.e., myocardial washout (WO). An abnormally increased ${ }^{123} \mathrm{I}-m \mathrm{IBG}$ WO has been associated with worsened patient outcome. ${ }^{4}$ While the exact mechanism of increased cardiac ${ }^{123} \mathrm{I}-m \mathrm{IBG}$ WO is not completely understood, Tamaki et al. ${ }^{17}$ did observe a significant correlation between $\mathrm{WO}$ and plasma NE concentration. This finding would be in accord with the concept that a potential mechanism of elevated WO is excessive local release of endogenous $\mathrm{NE}$, a reflection of increased sympathetic tone to the heart, competing with radiotracer for uptake sites ${ }^{16}$ although other factors likely also contribute. ${ }^{4,15}$

Another issue to consider is that RDN, by altering efferent innervation of the kidney, impedes output of renin, thus decreasing the production of RAAS neurohormones. Many studies have shown that medications directed at RAAS components, such as angiotensinconverting enzyme inhibitors, angiotensin receptor blocking agents, and mineralocorticoid blockers, can increase HMR and decrease WO. ${ }^{4}$ It is unclear how much of the image changes brought on by these medications is related to cardiac structural changes that impact local sympathetic nerve function versus hemodynamic improvements mediating changes to cardiac sympathetic drive.

Thus, numerous factors can contribute to observations and measurements from cardiac ${ }^{123} \mathrm{I}-m \mathrm{IBG}$ imaging, making it difficult to predict how such images would be affected by RDN. In the first reported study assessing the effect of RDN on cardiac ${ }^{123} \mathrm{I}-m \mathrm{IBG}$ imaging, Donazzan et al. ${ }^{18}$ assessed eleven patients with resistant hypertension just before and 6 months following RDN. Delayed HMR that was normal at baseline $(2.52 \pm 0.47)$ was essentially unchanged by the RDN. However, the mean baseline WO of $24.1 \pm 12.7 \%$ decreased to $7.9 \pm 25.5 \% \quad(P=0.047)$. While RDN succeeded in improving BP, the change in BP did not correlate with WO improvement.

\section{RDN AND ADRENERGIC IMAGING FOR CORONARY VASOSPASTIC ISCHEMIA}

Cardiac ${ }^{123} \mathrm{I}-m \mathrm{IBG}$ abnormalities have been reported in patients with vasospastic angina (VSA). Inobe et al. ${ }^{19}$ found regional tracer uptake reduction and abnormally high regional WO that were associated with more frequent anginal attacks and life-threatening ventricular arrhythmias. Watanabe et al. ${ }^{20}$ also found focal ${ }^{123} \mathrm{I}-m$ IBG abnormalities in patients with VSA. However, it is unclear whether abnormal cardiac sympathetic image findings in VSA are caused by ischemic myocardial damage, versus abnormalities of the sympathetic innervation shown by the images being the primary problem and then causing the VSA. In contrast to other reports, Sakata et al. ${ }^{21}$ described reduced regional WO in VSA patients compared with normal controls. As another report by this group ${ }^{22}$ showed that administration of atropine increased WO, the authors attributed the reduced washout associated with coronary spasm to potentially reflect enhanced parasympathetic tone. On the contrary, Taki et al. ${ }^{23}$ showed increased regional WO in patients with vasospastic angina, attributed to differences in disease severity and methods of analysis. Thus, for sure, the meaning of ${ }^{123} \mathrm{I}-m \mathrm{IBG}$ abnormalities in the setting of VSA for which RDN is employed as a potential therapy is difficult to predict. Of note, the ${ }^{123} \mathrm{I}-m \mathrm{IBG}$ abnormalities reported in these studies were focal, and thus tomographic imaging may be absolutely essential for proper evaluation of VSA using adrenergic imaging.

In the current issue of the Journal of the Nuclear Cardiology, Feyz and associates, ${ }^{24}$ knowing that the autonomic nervous system-sympathetic and parasympathetic components-is a key etiologic factor in VSA, seek to explore the effect of RDN both in terms of subjective clinical response and objectively measured changes in cardiac ${ }^{123} \mathrm{I}-m \mathrm{IBG}$ images. The authors cite a previous case report of theirs demonstrating resolution of dangerous ventricular arrhythmias following RDN, attributed to amelioration of hyperactive cardiac sympathetic activity in a patient with vasospastic angina. ${ }^{25}$

In their study, 10 subjects with refractory VSA underwent RDN using ultrasound emissions. Cardiac planar ${ }^{123}$ I- $m$ IBG imaging (but no SPECT), was performed at baseline and 6 months post RDN, and clinical response was assessed based on changes in Canadian Cardiovascular Society grading, BP and heart rate changes, and quality of life using the Seattle Angina Questionnaire. The authors hypothesized that with clinical improvement from relief of the VSA, ${ }^{123} \mathrm{I}-m \mathrm{IBG}$ 


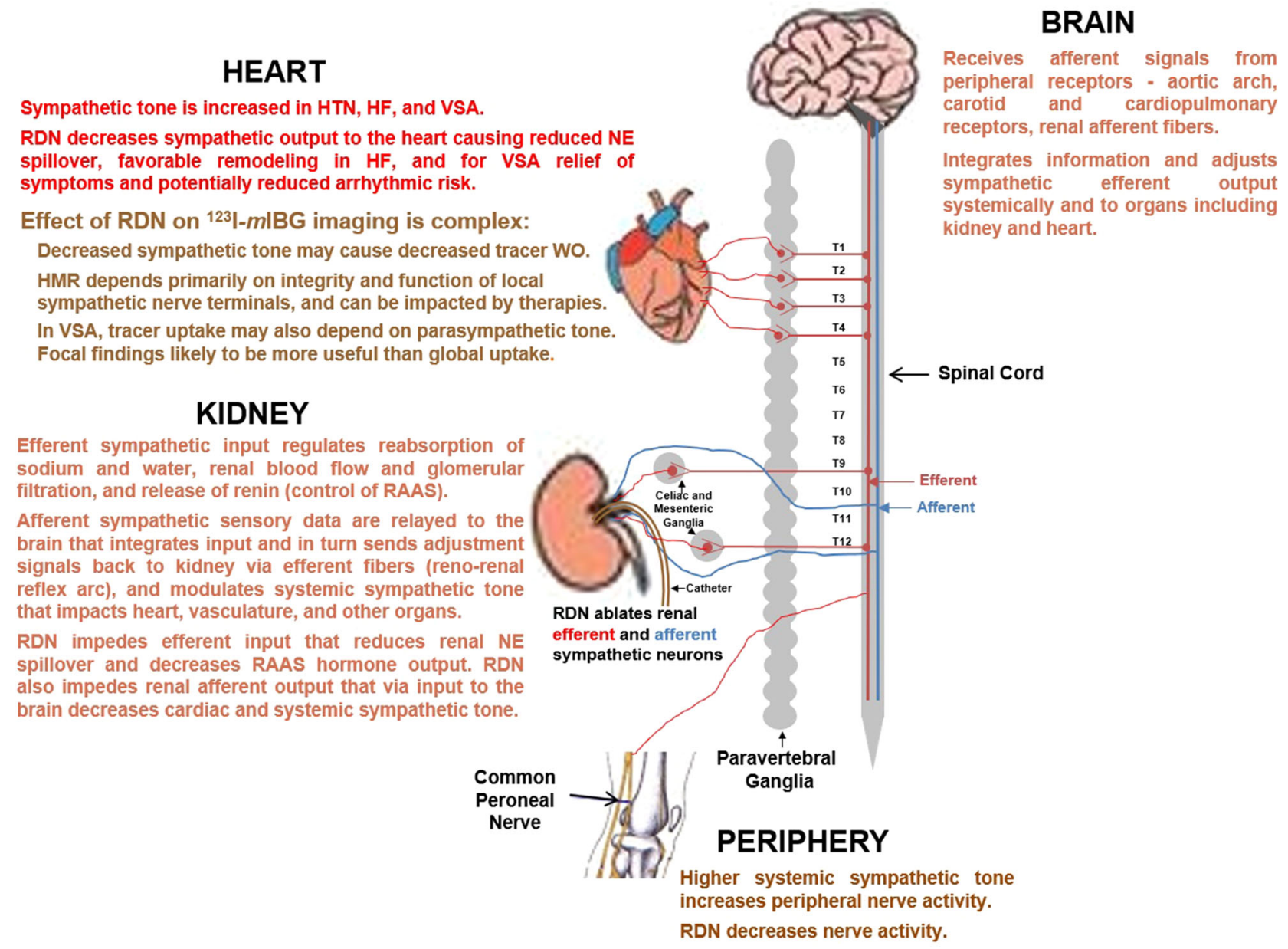

Figure 1. Schematic of selected components of cardiac, kidney, and systemic sympathetic innervation and tone, with potential effects on cardiac radionuclide adrenergic findings. (Note: the figure is a simplification of an extensively more complex system that also involves other organs, with some anatomic aspects not displayed in exact detail). $H F$, heart failure; $H M R$, heart-tomediastinum ratio; HTN hypertension; ${ }^{123} I-m I B G$, iodine-123 meta-iodobenzylguanidine; NE, norepinephrine; $R A A S$, renin-angiotensin-aldosterone system; $R D N$, renal denervation; $T$, thoracic; $V S A$, vasospastic angina; $W O$, washout.

parameters would improve as demonstrated by an increase in HMR and a decrease in washout.

As expected, the patients' clinical conditions improved with lower office BPs, fewer angina attacks, and less physical limitations, accompanied by better quality of life (although statistical power was limited by there being too few patients). ${ }^{123} \mathrm{I}-m \mathrm{IBG}$ image changes, however, were less impressive and of less clear meaning. No significant change was observed in either the initial or delayed median HMRs, with 4 patients showing an increase in delayed HMR, and 6 patients showing a decrease. With only 10 subjects, one cannot make conclusions on this matter. For WO, the number of patients ( 5 shown) was too underpowered for any meaningful conclusion, with 4 of the 5 patients increasing WO but the median value decreased from 15 to 13 because of one patient. It would have been helpful to have known if there were any changes in regional ${ }^{123} \mathrm{I}-m \mathrm{IBG}$ uptake WO given prior reports using tomographic imaging. Also to be considered, despite the diagnosis of VSA, the subjects in this investigation had baseline ${ }^{123} \mathrm{I}-m \mathrm{IBG}$ parameters within the normal range, making it difficult to show any image improvement with a therapy. As the authors propose, the investigation can be considered to be a pilot study that is hypothesis generating. 


\section{CONCLUDING THOUGHTS}

The autonomic nervous system is extremely complex, with selected aspects of the discussed interaction of the sympathetic components among the heart, kidney, brain, and periphery displayed and summarized in the schematic Figure 1. Sympathetic innervation plays an integral role in cardiovascular health, while disruption of it from disease leads to morbidity and increased mortality. While there are many methods to assess the system, cardiac radionuclide adrenergic imaging with ${ }^{123} \mathrm{I}-m$ IBG and analogous PET tracers is a relatively simple, quick, and noninvasive method of assessment of the cardiac component, with robust literature proving its risk stratification value. Better understanding of how cardiac adrenergic imaging can help assess the utility of therapies directed at extracardiac organ systems, such as renal denervation, should provide better understanding of disease processes in order to direct therapies that can improve outcome and well-being.

\section{Disclosure}

The author has no conflicts of interest.

\section{References}

1. Triposkiadis F, Karayannis G, Giamouzis G, Skoularigis J, Louridas G, Butler J. The sympathetic nervous system in hear failure. Physiology, pathophysiology and clinical implications. J Am Coll Cardiol 2009;54:1747-62.

2. Esler M, Lambert G, Rocca BL, Vaddadi G, Kaye D. Sympathetic nerve activity and neurotransmitter release in humans: Translation from pathophysiology into clinical practice. Acta Physiol 2003;177:275-84.

3. Vallbo AB, Hagbarth K, Wallin BG. Microneurography: how the technique developed and its role in the investigation of the sympathetic nervous system. J Appl Physiol 2004;96:1262-9.

4. Travin MI. Cardiac autonomic imaging with SPECT tracers. J Nucl Cardiol 2013;20:128-43.

5. Thackeray JT, Bengel FM. Assessment of cardiac autonomic neuronal function using PET imaging. $J$ Nucl Cardiol 2013;20:150-65.

6. Jacobson AF, Senior R, Cerqueira MD, Wong ND, Thomas GS, Lopez VA, Agostini D, Weiland F, Chanda H, Narula J, on behalf of the ADMIRE_HF Investigators. Myocardial iodine-123 metaiodobenzylguanidine imaging and cardiac events in heart failure. Results of the prospective ADMIRE-HF (AdreView Myocardial Imaging for Risk Evaluation in Heart Failure) study. J Am Coll Cardiol 2010;55:2212-21.

7. Travin MI, Matsunari I, Thomas GS, Nakajima K, Yoshinaga K. How do we establish cardiac sympathetic nervous system imaging with 123I-mIBG in clinical practice? Perspectives and lessons from Japan and the US. J Nucl Cardiol 2018. https://doi.org/10. 1007/s12350-018-1394-5.

8. Schlaich MP, Krum H, Sobotka PA, Esler M. Renal denervation and hypertension. Am J Hypertens 2011;24:635-42.
9. Sata Y, Head GA, Denton K, May CN, Schlaich MP. Role of the sympathetic nervous system and its modulation in renal hypertension. Front Med (Lausanne) 2018;5:82. https://doi.org/10. 3389/fmed.2018.00082.

10. Krum H, Schlaich M, Whitbourne R, Sobotka PA, Sadowski J, Bartus K, Kapelak B, Walton A, Sievert H, Thambar S, Abraham WT, Esler M. Catheter-based renal sympathetic denervation for resistant hypertension: A multicenter safety and proof-of-principle cohort study. Lancet 2009;373:1275-81.

11. Mauri L, Kario K, Basile J, Daeman J, Davies J, Kirtane AJ, et al. A multinational clinical approach to assessing the effectiveness of catheter-based ultrasound renal denervation: The RADIANCEHTN and REQUIRE clinical study designs. Am Heart J 2018;195:115-29.

12. Hering D, Marusic P, Walton AS, Lambert EA, Krum H, Narkiewicz K, Lambert GW, Esler MD, Schlaich M. Sustained sympathetic and blood pressure reduction 1 year after renal denervation inpatients with resistant hypertension. Hypertension 2014;64:118-24.

13. Brandt MC, Mahfoud F, Reda S, Schirmer SH, Erdmann E, Böhm M, Hoppe UC. Renal sympathetic denervation reduced left ventricular hypertrophy and improves cardiac function in patients with resistant hypertension. J Am Coll Cardiol 2012;59:901-9.

14. Fallavollita JA, Canty JM. Dysinnervated but viable myocardium in ischemic heart disease. J Nucl Cardiol 2010;17:1107-15.

15. Bengel FM. Imaging targets of the sympathetic nervous system of the heart: translational considerations. J Nucl Med 2011;52:116770.

16. Schofer J, Spielmann R, Schuchert A, Weber K, Schlüter M. Iodine-123 meta-iodobenzylguanidine scintigraphy: A noninvasive method to demonstrate myocardial adrenergic nervous system disintegrity in patients idiopathic dilated cardiomyopathy. J Am Coll Cardiol 1988;12:1252-8.

17. Tamaki S, Yamada T, Okuyama Y, Morita T, Sanada S, Tsukamoto Y, Masuda M, Okuda K, Iwasaki Y, Yasui T, Hori M, Fukunami M. Cardiac iodine-123 Metaiodobenzylguanidine imaging predicts sudden cardiac death independently of left ventricular ejection fraction in patients with chronic heart failure and left ventricular systolic dysfunction: Results from a comparative study with signal-averaged electrocardiogram, heart rate variability, and QT dispersion. J Am Coll Cardiol 2009;53:426-35.

18. Donazzan L, Mahfoud F, Ewen S, Ukena C, Cremers B, Kirsch C, Hellwig D, Eweiwi T, Ezziddin S, Esler M, Böhm M. Effects of catheter-based renal denervation on cardiac sympathetic activity and innervation in patients with resistant hypertension. Clin Res Cardiol 2016;105:364-71.

19. Inobe $\mathrm{Y}$, Kugiyama K, Miyagi H, Ohgushi M, Tomiguchi S, Takahashi M, Yasue H. Long-lasting abnormalities in cardiac sympathetic nervous system in patients with coronary spastic angina: Quantitative analysis with iodine 123 metaiodobenzylguanidine myocardial scintigraphy. Am Heart J 1997;134:112-8.

20. Watanabe K, Takahashi T, Miyajima S, Hirokawa Y, Tanabe N, Kato K, Kodama M, Aizawa Y, Tazawa S, Inoue M. Myocardial sympathetic denervation, fatty acid metabolism, and left ventricular wall motion in vasospastic angina. J Nucl Med 2002;43:147681.

21. Sakata K, Shirotani M, Yoshida H, Kurata C. Iodine-123 metaiodobenzylguanidine cardiac imaging to identify and localize vasospastic angina without significant coronary artery narrowing. J Am Coll Cardiol 1997;30:370-6.

22. Sakata K, Yoshida H, Hoshino T, Kurata C. Sympathetic nerve activity in the spasm-induced coronary artery region associated with disease activity of vasospastic angina. J Am Coll Cardiol 1996;28:460-4. 
23. Taki J, Yasuhara S, Takamatsu T, Nakajima K, Tatami R, Ishise S, et al. Value of iodine-123 metaiodobenzylguanidine scintigraphy in patients with vasospastic angina. Eur J Nucl Med 1998;25:229. 34

24. Feyz L, Henneman M, Verzijlbergen F, Kardys I, van Mieghem N, Daemen J. Renal sympathetic denervation in patients with vasospastic angina. J Nucl Cardiol 2019.
25. Feyz L, Wijchers S, Daemen J. Renal denervation as a treatment strategy for vasospastic angina induced ventricular tachycardia. Neth Heart J 2017;25:596-7.

Publisher's Note Springer Nature remains neutral with regard to jurisdictional claims in published maps and institutional affiliations. 